faser kann bei unserer mangelhaften Kenntniss dieser Eiweissstoffe nicht zur Zufriedenheit erklärt werden. In einer früheren Mittheilung ${ }^{1}$ ) schrieb ich die in der Faser statfindende Lackbildung der Wirkung der Lanugineäure zu. $O \mathrm{~b}$ sich während des Färbeprocesses dieses lösliche Abbau-Product in genügender Menge bilden kann, um die Maximal-Mengen der untersuchten Farbstoffe zu binden, ist zweifelhait, jedoch ist es nicht ausgeschlossen, dass dabei andere reactionsfähige Abbauproducte entstehen sollten, die zwischen dem löslichen Proteïl und der Wollsubstanz liegen, welche die Färbung bedingen.

Der Umstand, dass die beim Färben verwendete Wassermenge zwischen ziemlich weiten Grenzen auf die bei den quantitativen Ausfürbungen erhaltenen Zahlen nicht von Belang ist, steht in directem Widerspruch zu einer reinen Lösungstheorie. Auch scheint die Löslichkeit des Farl,stoffs in Wasser keinen Finfluss auf die ron der Faser aufgenommene Farbstoffmenge auszuïben.

Für die Ausarbeitung der meisten in dieser Abhandlung erwähnten Bestimmungen spreche ich hiermit Frl. Eva IIibbert meinen Dank aus.

Manchester, August 1904, Municipal School of Technology.

539. Franz Kuncke1l: Ueber einige Nitro-halogen- und Nitro-amino-Benzophenone.

[Mittheilıng ans dem chem. Univorsitäts-Laboratorium zu Rostock.]

(Eingegangen am 15. August 1904.)

Durch eine Publication ron $F$. Consonno ${ }^{2}$ ) sebe ich mich veranlasst, kurz über einige Benzophenonderivate, die ich mit Hrn. cand. chem. L. Szulc hergeste:It habe, zu berichten.

Erhitzt man 3 g 3.3'-Dibrombenzophenon (Schmp. 1410) mit ungefähr $20 \mathrm{ccm}$ rauchender Salpetersäure, bis keine branen Dämpfe mehr entweichen, und giesst alsdann die abgekühlte Lösung in viel Wasser, so erhält man das 3.3'- Dibrom-dinitrobenzophenon, $\mathrm{NO}_{z}$. $\mathrm{C}_{6} \mathrm{H}_{3} \mathrm{Br}$.CO. $\mathrm{C}_{6} \mathrm{H}_{3} \mathrm{Br} . \mathrm{NO}_{2}$, als gelbe, zähe Masse. Zur Reinigung zerreibt wan das Rohproduct mit heissem Alkohol uud krystallisirt aus Benzol um. Nach zweimaligem Krystallisiren schied sich das reine Dinitroderivat in blassgelben, derben Krystallen aus, die bei $20 \mathrm{~g}^{\mathrm{n}}$ schmelzen. Ausbeute ungefähr $60 \mathrm{pCt}$. der Theorie.

$0.2519 \mathrm{~g}$ Sbst: $0.3379 \mathrm{~g} \mathrm{CO}_{2}, 0.0432 \mathrm{~g} \mathrm{H}_{2} \mathrm{O} .-0.2559 \mathrm{~g}$ Slust.: $14.2 \mathrm{ccm}$ $\mathrm{N}\left(16^{0}, 762 \mathrm{~mm}\right)$.

1) loc. cit. 3) Gaz. chim. ital. 34 [I] 374-38i. 
$\mathrm{C}_{13} \mathrm{H}_{6} \mathrm{Br}_{2} \mathrm{~N}_{2} \mathrm{O}_{5}$. Ber. C 36.2, $\mathrm{H} 1.4, \mathrm{~N} 6.5$.

Gef. » 36.1 , 1.8 , 》 6.45 .

3-Brom-3'-amino-dinitrobenzophenon, $\mathrm{NO}_{2} \cdot \mathrm{C}_{6} \mathrm{H}_{3} \mathrm{Br}$. CO. $\mathrm{C}_{6} \mathrm{H}_{3}\left(\mathrm{NO}_{2}\right) . \mathrm{NH}_{2}$.

1 g 3.3'-Dibrom-dinitrobenzophenon wurde mit 4 ccm 25-procentigem, wässrigem Ammoniak und 4 ccrn Alkohol jm Bombenrohr 12 Stunden auf $130^{\circ}$ erhitzt. Hierauf wurde die gelbe Lösung auf dem Wasserbade eingedampft und der Rückstand mit Wasser auggewaschen. Aus Alkohol schied sich die Bromaminoverbindung in gelben, glänzenden Blättchen aus. Schmp. 250". Ausbeute fast quantitativ. Die Base löst sich schwer in starker Salzsäure.

$0.2455 \mathrm{~g}$ Sbst.: $25.4 \mathrm{ccm} \mathrm{N}\left(21^{0}, 768 \mathrm{~mm}\right)$.

$\mathrm{C}_{13} \mathrm{H}_{8} \mathrm{Br}_{5} \mathrm{~N}_{3}$. Ber. $\mathrm{N} 11.5, \mathrm{Br} 21.8$.

Gef. 》11.9, 》22.4.

\section{3'-Dipiperidino-dinitrobenzophenon, $\left(\mathrm{C}_{5} \mathrm{H}_{10} \mathrm{~N}\right)\left(\mathrm{NO}_{2}\right) \mathrm{C}_{6} \mathrm{H}_{3} \cdot \mathrm{CO} \cdot \mathrm{C}_{e} \mathrm{H}_{3}\left(\mathrm{NO}_{2}\right)\left(\mathrm{NC}_{5} \mathrm{H}_{10}\right)$.}

Leichter als Ammoniak wirkt Piperidin unter Ersatz der beiden Bromatome auf das 3.3'-Dibromdinitrobenzophenon ein. Durch 12. stündiges Erbitzen von $1 \mathrm{~g}$ Keton mit $2 \mathrm{~g}$ Piperidin und $5 \mathrm{ccm}$ Alkohol im Bombenrohr auf $125^{\circ}$ erbält man fast quantitative Umsetzung. Die Substanz löst sich schwer in Alkohol und Eisessig, leicht aber in Chloroform. Schmp. 190 . Aus Alkohol krystallisirt sie in kleinen, gelben Nädelchen, aus Eisessig dagegen in gelben Blättchæn.

$0.1196 \mathrm{~g}$ Sbst.: $13.1 \mathrm{cem} \mathrm{N}\left(21^{0}, 762 \mathrm{~mm}\right)$.

$\mathrm{C}_{23} \mathrm{~B}_{26} \mathrm{~N}_{4} \mathrm{O}_{5}$. Ber. $\mathrm{N}$ 12.7. Gef. $\mathrm{N}$ 12.5.

\section{3'-Dibrom-benzophenon, $(\mathrm{Br})^{4} \mathrm{C}_{6} \mathrm{H}_{4}$. CO. $\mathrm{C}_{6} \mathrm{H}_{4}(\mathrm{Br})^{3}$.}

Dieses Dibromderivat entsteht durch Erhitzen von $p$-Brombenzo. phenon mit Brom und Wasser im zugeschmolzenen Rohr auf $160^{\circ}$. Aus Alkohol scheidet es sich schwach rosa gefärbt aus und schmilzt bei $130^{\circ}$. Behandelt man es nach oben angegebener Weise mit rauchender Salpetersäure, so erhält man das

\section{3'Dibrom-dinitrobenzophenon.}

Gelbliche Krystalle aus Benzol und Eisessig; Schmp. 181 ${ }^{\circ}$. Au3beute gat.

$0.2009 \mathrm{~g}$ Sbst.: $0.1773 \mathrm{~g} \mathrm{AgBr}$.

$\mathrm{C}_{13} \mathrm{H}_{6} \mathrm{Br}_{2} \mathrm{~N}_{2} \mathrm{O}_{3}$. Ber. Br 37.2. Gef. Br 37.5.

Das in 4-Stellung befindliche Bromatom ist leichter beweglich als das in $3^{\prime}$ stehende. Erhitzt man nämlich $1 \mathrm{~g}$ des Dibromderivats mit $4 \mathrm{ccm}$ starkem, wässrigem Ammoniak und wenig Alkohol 12 Stunden auf $130^{\circ}$, so wird nur das in $4^{\prime}$ stehende Halogenatom durch Amid 
ersetzt. Das 4'-Amino-3'-brom-dinitrobenzophenon schmilzt bei $\geq 40^{\circ}$ und krystallisirt ans Alkohol in gelben Nädelchen; das Product löst sich schwer in Alkohol und Eisessig und ist unlöslich in Chloroform.

$0.1620 \mathrm{~g}$ Sbst.: $16.5 \mathrm{ccm} \mathrm{N}\left(20^{\prime \prime}, 761 \mathrm{~mm}\right) .-0.2376 \mathrm{~g}$ Sbst.: $0.12 \pm 8 \mathrm{~g}$ $\mathrm{A}: \mathrm{Br}$.

$$
\begin{array}{ll}
\mathrm{C}_{13} \mathrm{H}_{8} \mathrm{~B}_{1} \mathrm{O}_{3} \mathrm{~N}_{3 .} . & \text { Ber. N 11.5, Br 21.8. } \\
& \text { Gef. " 11.6, " } 21.9 .
\end{array}
$$

Um zu beweisen, dass vor das in 4 stehende Bromatom rengirt hatte, habe ich Ammoniak in derselben Weise auf 4-Chlor-3'brom-dinitrobenzophenon (Schmp. 1650) einwirken lassen. Hierbei erhielt ich dieselbe Verbindung.

$$
\begin{aligned}
& \text { 4-Piperidino-3'-brom-diuitrobenzophenon, } \\
& \left(\mathrm{C}_{6} \mathrm{H}_{10} \mathrm{~N}\right)^{1}\left(\mathrm{NO}_{2}\right) \mathrm{C}_{6} \mathrm{H}_{3} . \mathrm{CO} \cdot \mathrm{C}_{6} \mathrm{H}_{3}\left(\mathrm{NO}_{2}\right)(\mathrm{Br})^{3} \text {, }
\end{aligned}
$$

rutsteht sowohl aus dem 4.3'-Dibrom-, als auch aus dem 4-Chlor3 brom-Dinitrobenzophenon.

Uebergiesst man die beiden Ketone mit Piperidin, so tritt unter Erwärmen sofort Reaction ein. $U m$ eine vollkommene Umsetzung zn rzielen, erhitzt man $1 \mathrm{~g}$ Nitrohalogenbenzophenon mit $2 \mathrm{~g}$ Piperidin find $4 \mathrm{ccm}$ Alkobol in gescblossenen Robr in Wasserbade (anch am Rückflusskühler vollzieht sich die Reaction), wüscht den Rückstand mit Wasser aus und krystallisirt aus Alkobol um. Gelbrothe, in Albohol und Chloroform lösliche Nadeln, die bei $76^{\circ}$ unter Zersetzung schmelzen.

$0.1860 \mathrm{~g}$ Sbst.: $16.5 \mathrm{~cm} \mathrm{~N}\left(22^{0}, 758 \mathrm{~mm}\right)$. $\mathrm{C}_{18} \mathrm{H}_{16} \mathrm{~N}_{3} \mathrm{Br} \mathrm{O}_{5}$. Ber. N 9.6. Gef. $\mathrm{N} 9.8$.

Dieselben Reactionen babe ich mit Phenyl-p-tolylketon, sowie mit s- und $p$-Benzoylbenzoësäure ausführen lassen. Ebenso babe ich oArridobenzö̈säure mit Erfolg atuf die angeführten Dinitrohalogenverlindungen einwirken lassen.

Rostock, den 13. August 1904.

540. R. Stollé und W. Möring: Ueber die Condensation von Aldehyden mit $p$-Diketohexamethylen.

(Eingegangen am 15. Augnst 1904.)

Benzaldehyd condensirt sich mit $p$-Diketohexamethylen in ätherischer Lösung unter dem Einfluss ron Salzsäuregas. Aus dem nach Verdunsten des Aethers verbleibenden, ron Chlorwasserstoff und Wasser befreiten Rückstand warde durch Destillation im Vacuum 